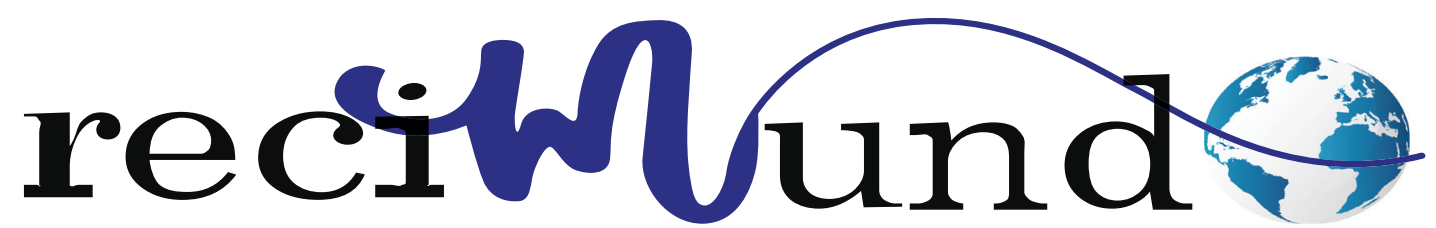

Revista Científica Mundo de la Investigación y el Conocimiento

DOI: 10.26820/recimundo/4.(1).enero.2020.416-422

URL: http://recimundo.com/index.php/es/article/view/766

EDITORIAL: Saberes del Conocimiento

REVISTA: RECIMUNDO

ISSN: 2588-073X

TIPO DE INVESTIGACIÓN: Artículo de Revisión

Código UNESCO: 3201 Ciencias Clínicas

PAGINAS: 416-422

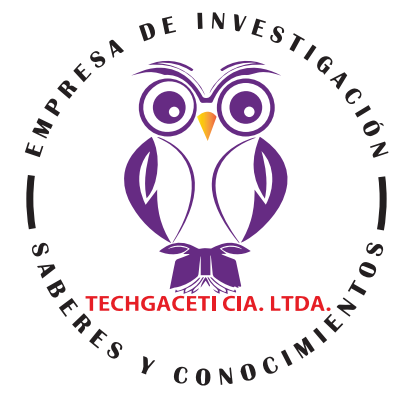

\title{
Riesgos quirúrgicos a pacientes diagnosticados con cuadro de peritonitis
}

\section{Surgical risks to patients diagnosed with peritonitis \\ Riesgos quirúrgicos para pacientes diagnosticados com padrão de peritonite}

\section{Edisson Javier Santana Bailón'; Cecilia Isabel Moyano Vega2; Diana Julissa Cevallos Vega3; Gladys Cecibel Avendaño Alonzo"; Jeniffer Maricruz Muentes Solórzano5; Eugenia Eunice Peralta Mogrovejo 6}

\section{RECIBIDO: 18/09/2019 ACEPTADO: 29/10/2019 PUBLICADO: 31/01/2020}

1. Médico Cirujano; Investigador Independiente; Manta, Ecuador; edisonsantanab@hotmail.es; (iD https://orcid. org/0000-0002-7934-5452

2. Médica Cirujana; Hospital de Especialidades Eugenio Espejo; Quito, Ecuador; cheoychabe@hotmail.com; (DD https://orcid.org/0000-0003-1757-6500

3. Médica Cirujana; Hospital Rodríguez Zambrano; Manta, Ecuador; diyucevaveg@hotmail.com; (D) https://orcid. org/0000-0001-8766-2653

4. Médico Cirujano; Hospital Rodríguez Zambrano; Manta, Ecuador; cecibelavend07@gmail.com; (iD https://orcid.org/0000-0002-5098-6782

5. Médico Cirujano; Hospital General IEES, Manta, Ecuador; jmmuentes@hotmail.com; (D) https://orcid.org/00000003-3768-3864

6. Médico Cirujano; Hospital General IEES, Manta, Ecuador; eugene_16@hotmail.es; (D) https://orcid.org/00000001-5954-7201

\section{CORRESPONDENCIA \\ Edisson Javier Santana Bailón \\ edisonsantanab@hotmail.es \\ Manta, Ecuador}




\section{RESUMEN}

El presente estudio examinó si la apendicitis aguda no perforada como emergencia quirúrgica requiere intervención inmediata y sus riesgos o es una enfermedad que puede ser tratada con una operación semielectiva. La apendicectomía inmediata ha sido el estándar de oro en el tratamiento de la apendicitis aguda, debido al riesgo de la progresión patológica. Sin embargo, esta práctica tradicional ha sido recientemente cuestionado por estudios que sugieren que las apendicectomías pueden ser electiva en algunos casos y todavía dar lugar a resultados positivos. Este fue un estudio retrospectivo de las historias clínicas de los pacientes que se sometieron a una apendicectomía para apendicitis aguda no perforada entre enero de 2007 y febrero de 2012. Los pacientes se dividieron en 2 grupos para la comparación: un grupo inmediato (los que fueron trasladados a una sala de operaciones dentro de las 12 horas después de la llegada al hospital) y un grupo retardada (aquellos entre 12 y 24 horas después de la llegada al hospital). Los puntos finales fueron la tasa de conversión, el tiempo quirúrgico, tasa de perforación, tasa de complicaciones, tasa de reingreso, la duración de la estancia hospitalaria y los costos médicos. No hubo diferencias significativas en la conversión abierta, el tiempo operatorio, perforación, las complicaciones postoperatorias, y la readmisión entre los 2 grupos.

Palabras clave: Apendicectomía, Apendicectomía Inmediata, Apendicitis no Perforada.

\section{ABSTRACT}

The present study examined whether acute non-perforated appendicitis as a surgical emergency requires immediate intervention and its risks or is a disease that can be treated with a semi-elective operation. Immediate appendectomy has been the gold standard in the treatment of acute appendicitis, due to the risk of pathological progression. However, this traditional practice has recently been questioned by studies that suggest that appendectomies may be elective in some cases and still lead to positive results. This was a retrospective study of the medical records of the patients who underwent an appendectomy for acute non-perforated appendicitis between January 2007 and February 2012. The patients were divided into 2 groups for comparison: an immediate group (those who were transferred to an operating room within 12 hours after arrival at the hospital) and a delayed group (those between 12 and 24 hours after arrival at the hospital). The end points were the conversion rate, surgical time, perforation rate, complication rate, readmission rate, length of hospital stay and medical costs. There were no significant differences in open conversion, operative time, perforation, postoperative complications, and readmission between the 2 groups.

Keywords: Appendectomy, Immediate Appendectomy, Non-Perforated Appendicitis.

\section{RESUMO}

O presente estudo examinou se a apendicite aguda não perfurada como emergência cirúrgica requer intervenção imediata e seus riscos ou é uma doença que pode ser tratada com uma operação semi-eletiva. A apendicectomia imediata tem sido o padrão-ouro no tratamento da apendicite aguda, devido ao risco de progressão patológica. No entanto, essa prática tradicional foi recentemente questionada por estudos que sugerem que apendicectomias podem ser eletivas em alguns casos e ainda levar a resultados positivos. Este foi um estudo retrospectivo dos prontuários médicos dos pacientes submetidos à apendicectomia por apendicite aguda não perfurada entre janeiro de 2007 e fevereiro de 2012. Os pacientes foram divididos em 2 grupos para comparação: um grupo imediato (aqueles que foram transferidos para um centro cirúrgico). quarto dentro de 12 horas após a chegada ao hospital) e um grupo atrasado (entre 12 e 24 horas após a chegada ao hospital). Os desfechos foram taxa de conversão, tempo cirúrgico, taxa de perfuração, taxa de complicações, taxa de readmissão, tempo de internação e custos médicos. Não houve diferenças significativas na conversão aberta, tempo operatório, perfuração, complicações pós-operatórias e readmissão entre os dois grupos.

Palavras-chave: Apendicectomia, Apendicectomia Imediata, Apendicite Não Perfurada. 


\section{Introducción}

La apendicitis aguda es una de las enfermedades más comunes que requieren una operación de emergencia. La apendicectomía inmediata se considera el estándar de oro del tratamiento para la apendicitis aguda. Se cree ampliamente que los retrasos en el diagnóstico y tratamiento contribuyen significativamente a una mayor incidencia de la apendicitis perforada, que se traducen en un aumento de la morbilidad del paciente (Berry \& Malt, 2004). Sin embargo, en algunos casos, la operación correspondiente se ha retrasado debido a razones como la falta de tiempo para el ayuno en la anestesia general, falta de disponibilidad de quirófanos, y overscheduling de los equipos operativos. Recientemente, algunos estudios han cuestionado el impacto de estos retrasos y nivel de atención con apendicectomía por lo que sugiere que la apendicitis o bien pueden ser tratada médicamente o utilizado en forma electiva sin aumentar la morbilidad.

Puede ocurrir en cualquier década de la vida, pero es más frecuente en los adolescentes y adultos jóvenes.

El tratamiento quirúrgico no está libre de complicaciones y éstos aumentan fuertemente la morbilidad asociada con el proceso, siendo los más comunes: Infección incisional con tasas que van desde 3.3 hasta $10.3 \%$ y las infecciones espacio de órganos $(9,4 \%)$. Hoy en día existe una gran variabilidad en el manejo quirúrgico y el resultado de AA basado en: abordaje quirúrgico y la técnica aplicada, el tipo de antibiótico utilizado, la duración de la antibioterapia, cierre de la herida quirúrgica, etc.

El diagnóstico de apendicitis aguda no perforada se basa en la decisión de un médico después de considerar manifestación clínica, examen físico, hallazgos de laboratorio y radiológicos. Los antibióticos como la cefalosporina se administran tan pronto como sea posible después del diagnóstico y se continúan hasta que el paciente descargue.

\section{Metodología}

Para el desarrollo de este proceso investigativo, se plantea como metodología la encaminada hacia una orientación científica particular que se encuentra determinada por la necesidad de indagar en forma precisa y coherente una situación, en tal sentido (Davila, 2015) define la metodología "como aquellos pasos previos que son seleccionados por el investigador para lograr resultados favorables que le ayuden a plantear nuevas ideas".(p.66)

Lo citado por el autor, lleva a entender que el desarrollo de la acción investigativa busca simplemente coordinar acciones enmarcadas en una revisión bibliográfica con el fin de complementar ideas previas relacionadas a los riesgos quirúrgicos en pacientes diagnosticados con cuadro de peritonitis a través de una revisión de literatura, para así finalmente elaborar un cuerpo de consideraciones generales que ayuden a ampliar el interés propuesto.

\section{Tipo de Investigación}

Dentro de toda práctica investigativa, se precisan acciones de carácter metodológico mediante las cuales, se logra conocer y proyectar los eventos posibles que la determinan, así como las características que hacen del acto científico un proceso interactivo ajustado a una realidad posible de ser interpretada. En este sentido, se puede decir, que la presente investigación corresponde al tipo documental, definido por Castro (2016), "se ocupa del estudio de problemas planteados a nivel teórico, la información requerida para abordarlos se encuentra básicamente en materiales impresos, audiovisuales y /o electrónicos". (p.41).

En consideración a esta definición, la orientación metodológica permitió la oportunidad de cumplir con una serie de actividades inherentes a la revisión y lectura de diversos documentos donde se encontraron ideas explicitas relacionadas con los tópicos encargados de identificar a cada caracterís- 
tica insertada en el estudio. Por lo tanto, se realizaron continuas interpretaciones con el claro propósito de revisar aquellas apreciaciones o investigaciones propuestas por diferentes investigadores relacionadas con el tema de interés, para luego dar la respectiva argumentación a los planteamientos, en función a las necesidades encontradas en la indagación.

\section{Fuentes Documentales}

El análisis correspondiente a las características que predomina en el tema seleccionado, llevan a incluir diferentes fuentes documentales encargadas de darle el respectivo apoyo y en ese sentido cumplir con la valoración de los hechos a fin de generar nuevos criterios que sirven de referencia a otros procesos investigativos. Para (CASTRO, 2016) las fuentes documentales incorporadas en la investigación documental o bibliográfica, "representa la suma de materiales sistemáticos que son revisados en forma rigurosa y profunda para llegar a un análisis del fenómeno".(p.41). Por lo tanto, se procedió a cumplir con la realización de una lectura previa determinada para encontrar aquellos aspectos estrechamente vinculados con el tema, con el fin de explicar mediante un desarrollo las respectivas apreciaciones generales de importancia.

\section{Técnicas para la Recolección de la Infor- mación}

La conducción de la investigación para ser realizada en función a las particularidades que determinan a los estudios documentales, tiene como fin el desarrollo de un conjunto de acciones encargadas de llevar a la selección de técnicas estrechamente vinculadas con las características del estudio. En tal sentido, (Bolívar, 2015), refiere, que es "una técnica particular para aportar ayuda a los procedimientos de selección de las ideas primarias y secundarias". (p. 71).

Por ello, se procedió a la utilización del subrayado, resúmenes, fichaje, como parte básica para la revisión y selección de los documentos que presentan el contenido teórico. Es decir, que mediante la aplicación de estas técnicas se pudo llegar a recoger informaciones en cuanto a la revisión bibliográfica de los diversos elementos encargados de orientar el proceso de investigación. Tal como lo expresa, (Bolívar, 2015) "las técnicas documentales proporcionan las herramientas esenciales y determinantes para responder a los objetivos formulados y llegar a resultados efectivos" (p. 58). Es decir, para responder con eficiencia a las necesidades investigativas, se introdujeron como técnica de recolección el método inductivo, que hizo posible llevar a cabo una valoración de los hechos de forma particular para llegar a la explicación desde una visión general.

Asimismo, se emplearon las técnicas de análisis de información para la realización de la investigación que fue ejecutada bajo la dinámica de aplicar diversos elementos encargados de determinar el camino a recorrer por el estudio, según, (Bolívar, 2015) las técnicas de procesamiento de datos en los estudios documentales "son las encargadas de ofrecer al investigador la visión o pasos que debe cumplir durante su ejercicio, cada una de ellas debe estar en correspondencia con el nivel a emplear" (p. 123). Esto indica, que para llevar a cabo el procesamiento de los datos obtenidos una vez aplicado las técnicas seleccionadas, tales como: fichas de resumen, textual, registros descriptivos entre otros, los mismos se deben ajustar al nivel que ha sido seleccionado.

\section{Resultados}

Se ha demostrado que la apendicectomías semielectiva para los pacientes con apendicitis aguda no perforada no aumentan la morbilidad (definida como la tasa de apertura de conversión, el tiempo operatorio, tasa de perforación, tasa de complicaciones postoperatorias, y la tasa de readmisión) pero aumentan los factores económicos, tales como los costos médicos y la duración 
SANTANA BAILÓN, E., MOYANO VEGA, C., CEVALlos VEGA, D., AVENDAÑo ALONZO, G., MUENTES SOLÓRZANO, J., \& PERALTA MOGROVEJO, E.

de la estancia hospitalaria.

En un estudio de 380 pacientes con apendicitis aguda, (Abou-Nukta \& Bakhos, 2016) ha demostrado que un retraso apendicectomía de más de 12 horas mostró un aumento no significativo de las tasas de perforación, el tiempo operatorio, o la duración de la estancia hospitalaria. Además, (Omundsen \&
Dennett, 2006) encontraron que no hubo diferencias en las tasas de complicaciones - la duración de la estancia hospitalaria postoperatoria entre los pacientes que fueron sometidos a apendicectomía dentro de 12 horas y de 12 a 24 horas después del ingreso.

Tabla 1. Precisión de la tomografía computarizada

\begin{tabular}{|c|c|c|c|c|c|}
\hline Variables & No perforada en la patología & Perforado en & logía & Todos & \\
\hline No perforada en la TC & $\begin{array}{r}879 \\
97,0 \% \text { un }\end{array}$ & $53,9 \%{ }^{\mathrm{C}}$ & 146 & 1025 & $\begin{array}{l}85,8 \%{ }^{\mathrm{mi}} \\
(879 / 1025)\end{array}$ \\
\hline Perforado en la TC & $\begin{array}{r}27 \\
3,0 \%{ }^{\mathrm{si}} \\
00 \%\end{array}$ & $46,1 \%{ }^{\mathrm{re}}$ & 125 & 152 & $82,2 \%^{\mathrm{F}}(125 / 152)$ \\
\hline
\end{tabular}

Los valores se presentan como el número a menos que se indique lo contrario.

$\mathrm{CT}$, tomografía computada.

${ }^{\text {un }}$ Sensibilidad; probabilidad de que los pacientes diagnosticados con apendicitis no perforada por CT entre los diagnosticados con apendicitis no perforada por la patología.

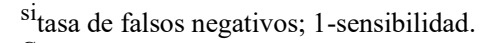

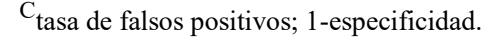

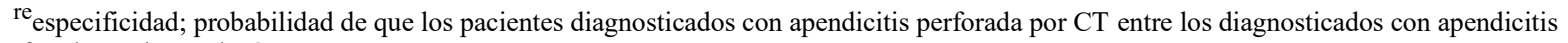
perforada por la patología.

${ }^{\mathrm{mi}}$ Valor predictivo positivo; probabilidad de que los pacientes diagnosticados con apendicitis no perforada por la patología entre los diagnosticados con apendicitis no perforada por la TC.

${ }^{\mathrm{F}} \mathrm{El}$ valor predictivo negativo; probabilidad de que los pacientes diagnosticados con apendicitis perforada por la patología entre los diagnosticados con apendicitis perforada por la TC.

Fuente: Autores 2020

Tabla 1. La exactitud de la ecografía

\begin{tabular}{|c|c|c|c|c|}
\hline Variables & No perforada en la patología & Perforado en la patología & Todos & \\
\hline No perforada en EE.UU. & $\begin{array}{r}530 \\
95,5 \% \text { un }\end{array}$ & $\begin{array}{l}58 \\
61,1 \% \mathrm{C}\end{array}$ & 588 & $\begin{array}{l}90,1 \%{ }^{\mathrm{mi}} \\
(530 / 588)\end{array}$ \\
\hline Perforado en EE.UU. & $\begin{array}{r}25 \\
4,5 \%\end{array}$ & $\begin{array}{l}37 \\
38,9 \% \text { re }\end{array}$ & 62 & $59,7 \%{ }^{\mathrm{F}}(37 / 62)$ \\
\hline Todos & 555 & 95 & 650 & \\
\hline
\end{tabular}

Los valores se presentan como números menos que se indique lo contrario.

Estados Unidos, la ecografía.

${ }^{\text {un }}$ Sensibilidad; probabilidad de los pacientes diagnosticados con apendicitis no perforada por los Estados Unidos entre los diagnosticados con apendicitis no perforada por la patología.

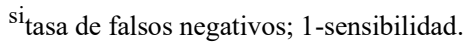

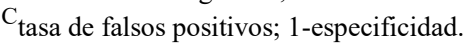

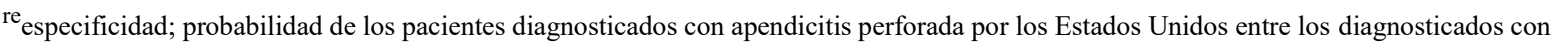
apendicitis perforada por la patología.

${ }^{\mathrm{mi}}$ Valor predictivo positivo; probabilidad de los pacientes diagnosticados con apendicitis no perforada por la patología entre los diagnosticados con apendicitis no perforada por nosotros.

$\mathrm{F}_{\mathrm{E}}$ valor predictivo negativo; probabilidad de pacientes con diagnóstico de apendicitis perforada por la patología entre los diagnosticados con apendicitis perforada por nosotros.

Fuente: Autores 2020 
En contraste con estos estudios, varios otros continúan apoyando el actual estándar de apendectomía como un procedimiento estándar de emergencia. En 1081 pacientes adultos con apendicitis aguda, (Ditillo \& Dziura, 2016) encontraron que el riesgo de desarrollar avanzadas patologías y complicaciones aumentaron con el tiempo hasta que los tratamientos apropiados, lo que sugiere que un retraso en la apendicectomía era inseguro. (Udgiri \& Curras, 2011) reportado que las tasas de complicaciones, duración de la estancia hospitalaria y los reingresos fueron mayores en un grupo apendicectomía retardada (realizado más de 10 horas después de la admisión) que en un grupo de apendicectomía inmediata (se realiza con menos de 10 horas después del ingreso) en un estudio de 211 pacientes con apendicitis. Recientemente, (Teixeira, 2012) mostró que mientras un retraso apendicectomía de más de 6 horas no aumentó el riesgo de perforación, aumentó significativamente el riesgo de infección del sitio quirúrgico en 4529 pacientes con apendicitis no perforada. En contraste, el presente estudio no mostró diferencias en la tasa de infección del sitio quirúrgico, que era aproximadamente el 5\% en cada grupo.

La seguridad del retraso de la apendicectomía se explica por el desarrollo de tecnologia médica, en particular la inyección de antibióticos para detener la progresión de la apendicitis. Un número de estudios han demostrado la eficacia de los antibióticos en el tratamiento de la apendicitis perforada. En la mayoría de los casos, conduce la administración de antibióticos a la resolución de los procesos infecciosos e inflamatorios de la apendicitis perforada, que permite apendicectomía electiva a realizar 6 a 8 semanas después de la presentación inicial de la enfermedad. Por otra parte, 2 ensayos controlados aleatorios sugirieron que la apendicitis aguda podría ser tratado con éxito con antibióticos y que los antibióticos pueden ser una terapia de primera línea en apendicitis aguda (Hansson \& Korner", 2009).
Los resultados de este informe pueden disminuir el estrés de los cirujanos en esta situación, ya que el aumento de riesgo de perforación y posterior morbilidad en la progresión de la apendicitis puede ser menos significativa que se pensaba. Este hallazgo optimista podría tener un efecto psicológico positivo en los cirujanos, lo que resulta en una operación más meticulosa al día siguiente con cuidado mejorado para los pacientes. Adicionalmente, la política actual que los especialistas cirujanos deben atender a sus pacientes en la sala de emergencias aumenta en gran medida la responsabilidad de los cirujanos. Estos hallazgos sugieren que los cirujanos podrían retrasar las operaciones para los pacientes con enfermedad menos crítica, tales como aquellos con apendicitis no perforada, con el fin de cuidar adecuadamente para aquellos que requieren atención inmediata, tales como los pacientes de trauma y pacientes de cuidados críticos, especialmente en situaciones con poco personal.

El diagnóstico preoperatorio preciso para aclarar si el apéndice está perforado debe ser un requisito previo para la apendicectomía diferida. La tomografía computarizada es una herramienta principal de diagnóstico con alta sensibilidad y especificidad para la apendicitis aguda. El uso rutinario de la TC en pacientes con sospecha de apendicitis aguda se ha demostrado acortar el tiempo necesario para la admisión sala de operaciones, reducir el número de apendicectomías negativas, y reducir los costos médicos (Rao \& Rhea, 2008). La ecografía es otra modalidad útil comúnmente usado para niños, pacientes embarazadas y pacientes ambulatorios, porque no es invasiva, no requiere de la preparación del paciente, y evita la exposición innecesaria a radiación ionizante. Por otra parte, (Peña, 2002) demostró que un protocolo de formación de imágenes usando Unidos y TC fue útil para distinguir entre apendicitis no perforado y perforado, como se muestra por una disminución marcada en la perforación 
y las tasas de apendicectomías negativas en 1338 niños con sospecha de apendicitis. Como las lecturas radiológicas no son infalibles, los cirujanos necesitan para confirmar la presencia de síntomas de perforación utilizando, exámenes físicos y de laboratorio. Los radiólogos también deben prestar mucha atención crítica a sus interpretaciones radiológicas.

\section{Conclusiones}

Para la apendicitis no complicada, se recomienda el alta temprana dentro de las 24 horas si no hay signos de complicación o no hay comorbilidades que puedan contraindicarlo. En la apendicitis complicada, la descarga variará según el progreso del paciente y la presencia de eventos adversos, pero en todos los casos se debe demostrar la restauración del tránsito intestinal y la ausencia de signos de complicación.

Se debe informar a los pacientes al alta sobre la atención que se debe seguir en el hogar y el monitoreo que se está recibiendo y se necesitará en los próximos días. La información adecuada, favorecida por el desarrollo de documentos estandarizados, reduce la tasa de readmisión y aumenta la satisfacción del paciente.

En conclusión, el retraso de la apendicectomía es seguro para los pacientes con apendicitis aguda no perforada. Se puede mejorar la calidad de la atención prestada a los cirujanos, mejorar la calidad del cuidado de los pacientes, y aumentar la utilización efectiva de los recursos médicos y salas de operación para emergencias que amenazan la vida.

\section{CITAR ESTE ARTICULO:}

Santana Bailón, E., Moyano Vega, C., Cevallos Vega, D., Avendaño Alonzo, G., Muentes Solórzano, J., \& Peralta Mogrovejo, E. (2020). Riesgos quirúrgicos a pacientes diagnosticados con cuadro de peritonitis. RECIMUNDO, 4(1), 416-422. doi:10.26820/recimundo/4.(1).enero.2020.416-422

\section{Bibliografía}

Abou-Nukta, F., \& Bakhos, C. (2016). Effects of delaying appendectomy for acute appendi-citis for 12 to 24 hours. Arch Surg, 506-507.

Berry, J. J., \& Malt, R. (2004). Appendicitis near its centenary. Ann Surg, 567-575.

Bolívar, J. (2015). Investigación Documental. México. Pax.

Castro, J. (2016). Técnicas Documentales. México. Limusa.

Davila, A. (2015). Concepto de terminos cientificos. Caracas: Oasis.

Ditillo, M., \& Dziura, J. (2016). Is it safe to delay appendectomy in adults with acute appendicitis? Ann Surg, 656-660.

Hansson, J., \& Korner", U. (2009). Randomized clinical trial of antibiotic therapy versus appendicectomy as primary treatment of acute appendicitis in unselected patients. Br J Surg, 473- 481.

Omundsen, M., \& Dennett, E. (2006). Delay to appendicectomy and associated morbidity: a retrospective review. . ANZ J Surg, 153-155.

Peña, B. (2002). Effect of an imaging protocol on clinical outcomes among pediatric patients with appendicitis. . Pediatrics, 1088-1093.

Rao, P., \& Rhea, J. (2008). Effect of computed tomography of the appendix on treatment of patients and use of hospital resources. N Engl J Med, 141146.

Teixeira, P. (2012). Appendectomy timing: waiting until the next morning increases the risk of surgical site infections. Ann Surg, 538-543.

Udgiri, N., \& Curras, E. (2011). Appendicitis, Is it an emergency? Am Surg, 898-901.

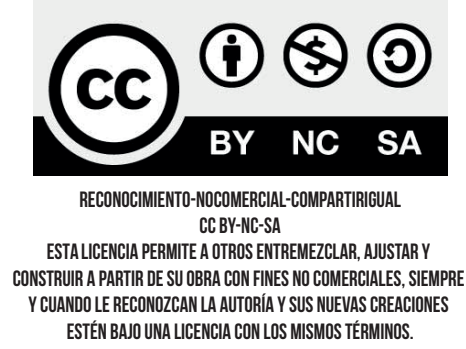

